\title{
Malignancy in Systemic Lupus Erythematosus
}

Giordano Egiziano, $M D^{1}$

Ann E. Clarke, MD, MSC ${ }^{2}$

Rosalind Ramsey-Goldman, MD, DrPh

Sasha Bernatsky, MD, PhD ${ }^{1,4, *}$

\author{
Address \\ ${ }^{1}$ McGill University, Montreal, QC, Canada \\ ${ }^{2}$ University of Calgary, Calgary, QC, Canada \\ ${ }^{3}$ Northwestern University, Chicago, IL, USA \\ ${ }^{*}, 4$ Division of Clinical Epidemiology, McGill University Health Center, 687 Pine \\ Avenue, V Building, Montreal, QC, H3A 1A1, Canada \\ Email: sasha.bernatsky@mcgill.ca
}

Published online: 28 January 2016

(C) Springer International Publishing AG 2016

This article is part of the Topical Collection on Lupus

Keywords Systemic lupus erythematosus (SLE) - Malignancy • Cancer • Lymphoma - Hematologic malignancies · Cyclophosphamide $\cdot$ Autoimmune dysregulation

\section{Opinion statement}

Systemic lupus erythematosus (SLE) is a multisystem inflammatory condition with an increasing number of new potential immunologic targets. These targets have shed light on both inflammatory and malignancy pathways given the interconnection between immunity and malignancy. In this review, we describe the various malignancies associated with SLE, as well as certain cellular pathways which contribute to dysregulated immunity in SLE and malignancy. This new appreciation for cancer risk in SLE adds yet another component to the management of SLE patients. Aside from managing the direct inflammatory consequences of SLE, part of a SLE patient's treatment should also include consideration of cancer risk profiles and knowing what types of cancer screening should be advocated. While current evidence for cancer screening in SLE is limited, cancer screening in SLE and other autoimmune conditions will likely be a growing focus of interest.

\section{Introduction}

Systemic lupus erythematosus (SLE) is an autoimmune inflammatory condition which can simultaneously affect multiple organ systems. Although SLE remains a very complex disease to treat, recent advances in therapies are prolonging life and clinicians are managing both traditional malignancy risk factors as well as treatment-related risk factors. Recent data have suggested that there is a slight but significant increased risk of overall malignancy in individuals with SLE compared to healthy controls $[1 \bullet \bullet]$. The specific types of malignancies that contribute to that increased risk include hematologic malignancies, lung cancer, and potentially 
other cancer types. Although there are interesting hypotheses as to why individuals with SLE have a higher risk of these cancers, it remains to be clarified to what extent cancer risk in SLE is altered by the disease process itself, as opposed to other factors, such as treatment. Altered cancer risk profiles in SLE are possibly due to both environmental and intrinsic dysregulation of immunity. As we describe below, it is becoming increasingly evident that there are common biological pathways in inflammatory diseases such as SLE and malignancies, some of which are also targets of novel treatments.

\section{Hematologic malignancies}

Hematologic malignancies (including lymphoma, leukemia, and multiple myeloma) have recently been shown to be increased in patients with SLE [1 $\bullet$, 2]. With the support of the Systemic Lupus International Collaborating Clinics (SLICC) research network, Bernatsky et al., using a large, multi-center, and international cohort, observed that the incidence and mortality of all hematologic malignancies was significantly elevated in SLE patients compared to the general population (standardized incidence ratio (SIR), 3.02; $95 \%$ confidence interval (CI), 2.48-3.63 and standardized mortality ratio (SMR), 2.8; $95 \% \mathrm{CI}$, $1.2-5.6)[1 \bullet \bullet]$. Similarly, a meta-analysis of five prospective cohort studies published between 2002 and 2013 also showed an increased risk of hematologic malignancy in SLE patients with a pooled SIR of 2.9; $95 \%$ CI, 2.0-4.4 [3]. Of the hematologic malignancies, the association was greatest for non-Hodgkin lymphoma (NHL) (SIR 5.7; $95 \%$ CI, 3.6-9.1) [3]. The most common type of NHL in SLE is diffuse large B cell lymphoma (DLBCL) [4]. DLBCL can be further divided into germinal center B cell-like (GCB) and non-GCB (or activated B cell-like). Preliminary evidence suggests that the non-GCB cell-oforigin DLBCL is more common in SLE, implying that lymphoma development in SLE and other autoimmune rheumatic diseases may be secondary to chronic immune stimulation [5].

Despite clear links between autoimmune conditions like SLE and lymphoma, we still do not understand the exact pathways by which these lymphomas

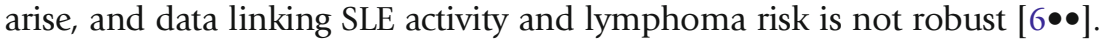
However, an important candidate pathway includes B cell-activating factor (BAFF) and a proliferation-inducing ligand (APRIL), inflammatory cytokines which promote B cell activation and contribute to disease activity in SLE [7, 8]. APRIL is strongly expressed in DLBCL in the general population and is detected in high concentrations in the sera of some patients with SLE, and these authors have also shown high expression of APRIL in DLBCL in SLE [9••]. Given the roles of BAFF and APRIL in lymphocyte activation, it is not surprising that these molecules are also implicated in the pathophysiology of hematologic malignancies, particularly in autoimmune diseases.

In addition to the innate pathways which may link malignancy with SLE, treatment-related effects may also contribute to overall malignancy risk. However, the direct impact of immunosuppressants on malignancy is not clear. In a recent case-cohort analysis within the SLICC multisite SLE cohort, there was suggestion of a greater lymphoma risk in SLE individuals exposed to cyclo-

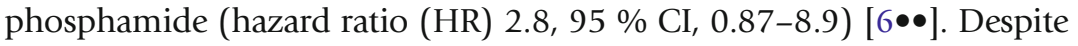
concern regarding malignancy risk in rheumatic diseases treated with 
immunosuppressants $[10,11]$, most cases of lymphoma in the above case-

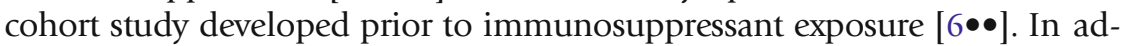
dition, it may be difficult to completely adjust for disease activity in these studies, which could also influence malignancy risk. In addition, the immunosuppressive actions of cyclophosphamide or other agents could promote the development of oncogenic viruses in SLE patients. In particular, Epstein-Barr virus (EBV), which is known to be implicated in the etiology of various hematologic malignancies, has been repeatedly investigated in SLE patients $[12,13]$. In a recent meta-analysis, EBV antiviral capsid antigen was significantly associated with SLE, supporting the hypothesis that EBV infection may predispose to the development of SLE [14] and potentially explain some of the increased lymphoma risk in SLE. However, despite certain case reports citing an association between immunosuppression and EBV in autoimmune conditions [15], larger population-based studies have failed to demonstrate a clear link between lymphoma and EBV in inflammatory diseases [16].

It is interesting that several common signaling pathways between SLE activity and hematologic malignancy are also targets for emerging SLE therapies. Currently, the monoclonal antibody belimumab, which inhibits circulating BAFF, is the only biologic agent specifically approved for the treatment of SLE [17-19]. Another potential therapeutic agent in SLE is atacicept, a fusion protein of a transmembrane activator and cyclophilin ligand interactor (TACI), which binds to and inhibits BAFF and APRIL receptors $[20,21]$. Given the emergence of such therapies, continued surveillance of lymphoma risk in SLE (and whether the profile of increased risk in SLE can actually be lowered by such agents) is of interest and warrants future study.

\section{Lung malignancies}

In 2012, Hemminki et al. demonstrated an increased incidence of lung cancer in SLE patients [22]. Using the Swedish Hospital Discharge Registry, they identified 7624 patients with SLE and concluded that the greatest incidence was seen in small cell lung cancer (SIR 3.38, 95 \% CI, 1.895.59), followed by squamous cell carcinoma, large cell carcinoma, and adenocarcinoma [22]. In addition to an increased incidence of cancer, small cell lung cancer in SLE patients was associated with a significant mortality risk (SMR 2.69, 95 \% CI 2.11-3.38). Although their analyses were adjusted for chronic obstructive pulmonary disease and socioeconomic status in an attempt to control for smoking habits, the possibility of confounding due to individual smoking patterns remains a concern. Most recently, in the largest-ever clinical SLE cohort study (SLICC study), the SIR for lung cancer was $1.30(95 \%$ CI 1.04, 1.60) $[1 \bullet \bullet]$ and a meta-analysis of seven cohort studies confirmed an increased risk of lung cancer in SLE patients (pooled SIR 1.68, $95 \%$ CI 1.33-2.13) [23].

Case-cohort analyses (nested with the SLICC cohort study) investigating the risk of lung cancer in SLE have suggested smoking as an important risk factor itself [24]. At the same time, there is evidence to suggest that smoking may predispose to increased disease activity in SLE [25]. 


\section{Breast cancer}

Preliminary updated analyses confirm this and did suggest a trend for greater cancer risk in SLE patients with higher cumulative disease activity over time (HR 1.81, 0.90, 3.63) although the CI included the null value [26]. The estimated adjusted effects of all drugs were relatively imprecise. Although some suspect interstitial lung disease as a risk factor for lung cancer [27], in a review of the lung cancer cases arising from the international SLE cohort study, none had pulmonary fibrosis recorded as a damage index item [28].

In addition to its association between inflammatory conditions and hematologic malignancies, IL- 6 has also been shown to be associated with lung manifestations in mouse models of SLE [29]. Similarly, the anti-IL-6 receptor antibody, tocilizumab, has been shown to induce apoptosis in non-small cell lung cancer [30]. Taken together, it is clear that the pathophysiology, which drives lung cancer risk in SLE, is likely a multifactorial combination of intrinsic and extrinsic risk factors.

A 2011 meta-analysis by Bernatsky et al. demonstrated a significant reduction in breast cancer risk in SLE patients (SIR 0.76, $95 \%$ CI 0.69-0.85) [31••]. The finding that hormone-driven malignancies such as breast cancer are reduced in SLE patients has also been demonstrated by various other studies $[1 \bullet \bullet, 32]$. Breast cancer risk was reduced in both pre- and post-menopausal women $[31 \bullet \bullet]$. A study using the Surveillance, Epidemiology and End ResultsMedicare-linked database in 1993-2002 showed a risk reduction specifically of estrogen receptor (ER)-negative breast cancer (odds ratio 0.49, 95 \% CI 0.260.93 ) among elderly women with SLE [33••]. Following up on this result, investigators found a decreased proportion of ductal subtypes of breast cancer in SLE; ductal breast cancers tend to be ER and progesterone receptor-negative [34•]. Analyses of a large breast cancer genome-wide association study did not demonstrate important associations between breast cancer and 10 lupusassociated SNPs [35]. The authors concluded that if decreased breast cancer risk in SLE is influenced by genetic profiles, this may be due to complex interactions and/or epigenetic factors.

\section{Cervical and vulvar cancer}

In contrast to breast cancer risk, Bernatsky et al. have shown an increased risk of vulvar cancers in SLE patients (SIR 3.78, $95 \%$ CI 2.41-4.31) [1••]. Similarly, multiple studies have demonstrated an increased risk of cervical dysplasia and of high-grade squamous intraepithelial lesions in SLE patients, which is potentially heightened by immunosuppressive drugs [36, 37]. The 2014 metaanalysis by Zard et al. showed a significant increase of high-grade squamous epithelial lesions in SLE patients compared to healthy age-matched controls (pooled OR 8.66, 95 \% CI 3.75-20) [37]. However, the results of this metaanalysis must be interpreted with caution as a significant proportion of patients were from countries where cervical screening is not systematically offered or available to patients [38]. In another study of a national Danish registry between 1977 and 2010, investigators assessed 5695 women with SLE. A total of 


\section{Liver cancer}

14 cervical cancer cases were observed, and the SIR for high-grade squamous intraepithelial lesions in SLE was 1.1, $95 \%$ CI 0.6-1.8) [38]. Of note, in the abovementioned study by Bernatsky et al., the risk of cervical cancer was unclear $[1 \bullet \bullet]$. A recent attempt to review cancer screening recommendations in SLE found no original research studies directly comparing cancer screening strategies in SLE, although, generally, authors recommend adherence to general population screening measures, particularly cervical screening [39].

Given the possible association between SLE and certain viral-driven diseases, the risk of hepatobiliary malignancies in SLE patients is another subject of interest. Various studies have documented an increased risk of hepatobiliary malignancies in SLE. The 2014 meta-analysis by Ni et al., which included five cohort studies, demonstrated an increased risk of hepatic cancers (SIR 2.44, $95 \%$ CI 1.46-4.05) [23]. In addition, a recent large cohort study from Sweden, which identified 6350 SLE patients through a national hospital discharge registry, found an increased risk of primary hepatic malignancies (SIR 4.17, $95 \%$ CI 2.42-6.69) [40]. Although the exact pathophysiology behind the increased risk of hepatic malignancy must still be determined, the overall risk of primary liver cancer in SLE may not be surprising, given the risk of overlap syndromes such as autoimmune hepatic disease, primary biliary cirrhosis, and a possible increased prevalence of hepatitis C infection in SLE [41].

Other malignancies of interest in SLE include bladder cancer, thyroid cancer, and prostate cancer. A 2014 meta-analysis by Zhang et al. which included seven cohort studies found a positive association between thyroid cancer and SLE patients (pooled SIR 2.22, $95 \%$ CI, 2.11-2.34) [42]. Although the underlying pathways have not been elucidated, it is reasonable to suspect autoimmune dysregulation in SLE patients as a potential risk factor for the development of thyroid malignancy.

The evidence for an increased risk of bladder cancer in SLE patients is less clear. Two large cohort studies have suggested an increased risk of bladder cancer in SLE $[36,43]$. A meta-analysis by Ni et al. included six cohort studies for bladder cancer and suggested a trend for increased bladder cancer in SLE patients (SIR 1.78, $95 \%$ CI, 0.85-3.72) [23]. However, the analysis by Dreyer et al. revealed that all SLE patients who developed bladder cancer had received cyclophosphamide [36]; hence, an increased risk of bladder cancer may not be due to SLE itself, but rather cyclophosphamide. In clinical SLE cohort studies, bladder cancer remains a very uncommon outcome.

In a meta-analysis providing a pool of 6068 male SLE patients observed for a total of 38,186 patient-years, the SIR estimate for prostate cancer risk in males with SLE, compared to the general population, was 0.72 (95\% CI 0.57, 0.89) [44•]. The authors point out that since alterations in androgen pathways can potentially alter prostate risk, the lower risk of prostate cancer in men with SLE could possibly be due to hypoadrenergic states (although this hypothesis has not been specifically tested). 


\section{Conclusions}

In this review of malignancies associated with SLE, the evidence from both individual cohort studies and meta-analyses suggests a slight overall increased risk of cancer in SLE patients compared to the general population (SIR 1.14, $95 \%$ CI, 1.05-1.23) [1••]. In particular, the risk of hematologic, pulmonary, hepatic, and thyroid cancers appears to be elevated in SLE, while the risk of breast and prostate cancers is reduced. As we have presented above, the pathophysiology underlying specific cancers may be a combination of dysregulated immune signaling and environmental insults, leading to molecular changes which affects both inflammatory and neoplastic pathways.

Given recent advances in the diagnosis and treatment of inflammatory conditions such as SLE, physicians need to consider various long-term risk factors and comorbidities in their patients. Clarification of the role of treatmentrelated effects in the development of malignancy in SLE will require more longterm studies.

With respect to cancer screening, this should be tailored to a patient's individual risk factor profile. For example, in SLE patients exposed to cyclophosphamide, there may be a role for more frequent pap smears or, as suggested by Tessier-Cloutier et al., there may be a role for urine cytology screening [39]. The controversial topic of low-dose CT screening for lung cancer is another concept which may eventually be applied to SLE patients with a smoking history [45]. While these concepts may sound intuitive, future studies will be required to assess whether such screening interventions translate into appropriate risk reduction while minimizing harm.

\section{Compliance with Ethical Standards}

\section{Conflict of Interest}

Giordano Egiziano declares that he has no conflict of interest. Ann E. Clarke declares that she has no conflict of interest. Rosalind Ramsey-Goldman declares that she has no conflict of interest. Sasha Bernatsky declares that she has no conflict of interest.

Human and Animal Rights and Informed Consent

This article does not contain any studies with human or animal subjects performed by any of the authors.

\section{References and Recommended Reading}

Papers of particular interest, published recently, have been highlighted as:

- Of importance

- Of major importance

1.• Bernatsky S, Ramsey-Goldman R, Labrecque J, et al. Cancer risk in systemic lupus: an updated international multicentre cohort study. J Autoimmun. 2013;42:130-5.

This international SLE cohort is the largest to date and demonstrated a small overall increased cancer risk in SLE patients versus the general population. In addition, there was a clear 
increased incidence of non-Hodgkin lymphoma, as well as cancers of the lung, vulva, and thyroid.

2. Parikh-Patel A, White RH, Allen M, Cress R. Cancer risk in a cohort of patients with systemic lupus erythematosus (SLE) in California. Cancer Causes Control. 2008;19(8):887-94.

3. Apor E, O'Brien J, Stephen M, Castillo JJ. Systemic lupus erythematosus is associated with increased incidence of hematologic malignancies: a meta-analysis of prospective cohort studies. Leuk Res. 2014;38(9):1067-71.

4. Bernatsky S, Ramsey-Goldman R, Rajan R, et al. NonHodgkin's lymphoma in systemic lupus erythematosus. Ann Rheum Dis. 2005;64(10):1507-9.

5. Tessier Cloutier B, Farinha P, Bernatsky S, Baecklund E, Clarke AE, Ramsey-Goldman R, Gascoyne R. Cell of origin of diffuse large B-cell lymphoma (DLBCL) in patients with systemic lupus erythematosus (SLE) [abstract]. Arthritis Rheumatol. 2015; 67 (suppl 10). http://acrabstracts.org/abstract/cell-of-origin-ofdiffuse-large-b-cell-lymphoma-dlbcl-in-patients-withsystemic-lupus-erythematosus-sle/. Accessed October 6, 2015.

6.• Bernatsky S, Ramsey-Goldman R, Joseph L, et al. Lymphoma risk in systemic lupus: effects of disease activity versus treatment. Ann Rheum Dis. 2014;73(1):138-42.

In this case-cohort analysis within a multisite SLE cohort, there was suggestion of increased lymphoma incidence in patients exposed to cyclophosphamide and to higher cumulative steroid doses. The contribution of disease activity to lymphoma risk was not clear in this study.

7. Stohl W. Therapeutic targeting of the BAFF/APRIL axis in systemic lupus erythematosus. Expert Opin Ther Targets. 2014;18(4):473-89.

8. Zollars E, Fang H, Bienkowska J, et al. Only BAFF mRNA, not BAFF protein level in blood, is associated with SLE activity over one year. Ann Rheum Dis. 2015;74:A74.

9.• Lofstrom B, Backlin C, Pettersson T, Lundberg IE, Baecklund E. Expression of APRIL in diffuse large B cell lymphomas from patients with systemic lupus erythematosus and rheumatoid arthritis. J Rheumatol. 2011;38(9):1891-7.

In this study, lymphoma biopsies from individuals with RA, SLE and control cases were stained to assess for expression of APRIL. A high expression of APRIL was detected in SLE lymphoma cases (OR 23.6, 95 \% CI 2.4-231.2), and Epstein-Barr virus in the lymphoma tissue was significantly associated with APRIL expression.

10. Jones M, Symmons D, Finn J, Wolfe F. Does exposure to immunosuppressive therapy increase the 10 year malignancy and mortality risks in rheumatoid arthritis? A matched cohort study. Br J Rheumatol.

1996;35(8):738-45.

11. Baker GL, Kahl LE, Zee BC, Stolzer BL, Agarwal AK, Medsger Jr TA. Malignancy following treatment of rheumatoid arthritis with cyclophosphamide. Longterm case-control follow-up study. Am J Med. 1987;83(1):1-9.
12. Draborg AH, Duus K, Houen G. Epstein-Barr virus in systemic autoimmune diseases. Clin Dev Immunol. 2013;2013:535738.

13. Rasmussen NS, Draborg AH, Nielsen CT, Jacobsen S, Houen G. Antibodies to early EBV, CMV, and HHV6 antigens in systemic lupus erythematosus patients. Scand J Rheumatol. 2014;44(2):143-9.

14. Hanlon P, Avenell A, Aucott L, Vickers MA. Systematic review and meta-analysis of the sero-epidemiological association between Epstein-Barr virus and systemic lupus erythematosus. Arthritis Res Ther.

2014;16(1):R3.

15. Kamel OW, Van de Rijn M, Hanasono MM, Warnke RA. Immunosuppression-associated lymphoproliferative disorders in rheumatic patients. Leuk Lymphoma. 1995;16(5-6):363-8.

16. Baecklund E, Iliadou A, Askling J, et al. Association of chronic inflammation, not its treatment, with increased lymphoma risk in rheumatoid arthritis. Arthritis Rheum. 2006;54(3):692-701.

17. Morais SA, Vilas-Boas A, Isenberg DA. B-cell survival factors in autoimmune rheumatic disorders. Ther $\mathrm{Adv}$ Musculoskelet Dis. 2015;7(4):122-51.

18. Navarra SV, Guzman RM, Gallacher AE, et al. Efficacy and safety of belimumab in patients with active systemic lupus erythematosus: a randomised, placebo-controlled, phase 3 trial. Lancet. 2011;377(9767):721-31.

19. Furie R, Petri M, Zamani O, et al. A phase III, randomized, placebo-controlled study of belimumab, a monoclonal antibody that inhibits B lymphocyte stimulator, in patients with systemic lupus erythematosus. Arthritis Rheum. 2011;63(12):3918-30.

20. Dall'Era M, Chakravarty E, Wallace D, et al. Reduced B lymphocyte and immunoglobulin levels after atacicept treatment in patients with systemic lupus erythematosus: results of a multicenter, phase $\mathrm{Ib}$, double-blind, placebo-controlled, dose-escalating trial. Arthritis Rheum. 2007;56(12):4142-50.

21. Isenberg D, Gordon C, Licu D, Copt S, Rossi CP, Wofsy D. Efficacy and safety of atacicept for prevention of flares in patients with moderate-to-severe systemic lupus erythematosus (SLE): 52-week data (APRIL-SLE randomised trial). Ann Rheum Dis. 2014.

22. Hemminki K, Liu X, Ji J, Sundquist J, Sundquist K. Effect of autoimmune diseases on risk and survival in histology-specific lung cancer. Eur Respir J. 2012;40(6):1489-95.

23. Ni J, Qiu LJ, Hu LF, et al. Lung, liver, prostate, bladder malignancies risk in systemic lupus erythematosus: evidence from a meta-analysis. Lupus. 2014;23(3):284-92.

24. Bernatsky S, Joseph L, Boivin JF, et al. The relationship between cancer and medication exposures in systemic lupus erythaematosus: a case-cohort study. Ann Rheum Dis. 2008;67(1):74-9.

25. Bourre-Tessier J, Peschken CA, Bernatsky S, et al. Association of smoking with cutaneous manifestations in systemic lupus erythematosus. Arthritis Care Res (Hoboken). 2013;65(8):1275-80. 
26. Bernatsky S R-GR, Petri M, Urowitz MB, Gladman DD, Yelin EH, et al. Lung Cancer in SLE (Abstract). Arthritis and Rheumatology 2015: S2791 http://fuldamedia. com/acr2015/abstract/lung-cancer-in-sle/ Accessed October 21, 2015.

27. Daniels CE, Jett JR. Does interstitial lung disease predispose to lung cancer? Curr Opin Pulm Med. 2005;11(5):431-7.

28. Bin J, Bernatsky S, Gordon C, et al. Lung cancer in systemic lupus erythematosus. Lung Cancer. 2007;56(3):303-6.

29. Maier-Moore JS, Horton CG, Mathews SA, et al. Interleukin-6 deficiency corrects nephritis, lymphocyte abnormalities, and secondary Sjogren's syndrome features in lupus-prone Sle1.Yaa mice. Arthritis Rheumatol. 2014;66(9):2521-31.

30. Kim NH, Kim SK, Kim DS, et al. Anti-proliferative action of IL-6R-targeted antibody tocilizumab for nonsmall cell lung cancer cells. Oncol Lett. 2015;9(5):2283-8.

31.• Bernatsky S, Ramsey-Goldman R, Foulkes WD, Gordon C, Clarke AE. Breast, ovarian, and endometrial malignancies in systemic lupus erythematosus: a meta-analysis. Br J Cancer. 2011;104(9):1478-81.

This meta-analysis which focused on female malignancies in SLE included data from five large SLE cohorts and strongly supported a decreased risk of breast, ovarian, and endometrial cancers in SLE.

32. Dey D, Kenu E, Isenberg DA. Cancer complicating systemic lupus erythematosus-a dichotomy emerging from a nested case-control study. Lupus. 2013;22(9):919-27.

33.• Gadalla SM, Amr S, Langenberg P, et al. Breast cancer risk in elderly women with systemic autoimmune rheumatic diseases: a population-based case-control study. Br J Cancer. 2009;100(5):817-21.

This population-based case-control study of elderly women (aged 67-99 years) found that women with rheumatoid arthritis were less likely to develop breast cancer (OR 0.87, $95 \%$ CI 0.82-0.93), and that women with SLE were less likely to develop an estrogen-receptor negative breast cancer (OR 0.49, $95 \%$ CI 0.26-0.93).

34. $\quad$ Tessier-Cloutier B, Wang YF, Labrecque J, et al. Breast cancer in systemic lupus erythematosus. J Rheumatol. 2013;40(6):993.

This study identified 180 breast cancer cases within a SLE cohort, with histological data available for 155 cases. While the majority of breast cancers were ductal carcinomas, there was suggestion that ductal carcinomas (which are typically estrogen-receptor negative) are decreased in SLE patients. This study also demonstrated that age and duration of SLE were independent predictors of histological type.

35. Bernatsky S, Easton DF, Dunning A, et al. Decreased breast cancer risk in systemic lupus erythematosus: the search for a genetic basis continues. Lupus. 2012;21(8):896-9.

36. Dreyer L, Faurschou M, Mogensen M, Jacobsen S. High incidence of potentially virus-induced malignancies in systemic lupus erythematosus: a long-term followup study in a Danish cohort. Arthritis Rheum. 2011;63(10):3032-7.

37. Zard E, Arnaud L, Mathian A, et al. Increased risk of high grade cervical squamous intraepithelial lesions in systemic lupus erythematosus: a meta-analysis of the literature. Autoimmun Rev. 2014;13(7):730-5.

38. Dugue PA, Lynge E, Rebolj M. Increased risk of highgrade squamous intraepithelial lesions in systemic lupus erythematosus: additional data from Denmark. Autoimmun Rev. 2014;13(12):1241-2.

39. Tessier-Cloutier B, Clarke AE, Ramsey-Goldman R, Gordon C, Hansen JE, Bernatsky S. Systemic lupus erythematosus and malignancies: a review article. Rheum Dis Clin North Am. 2014;40(3):497-506. viii.

40. Castro FA, Liu X, Forsti A, et al. Increased risk of hepatobiliary cancers after hospitalization for autoimmune disease. Clin Gastroenterol Hepatol. 2014;12(6):1038-45 e7.

41. Bessone F, Poles N, Roma MG. Challenge of liver disease in systemic lupus erythematosus: clues for diagnosis and hints for pathogenesis. World J Hepatol. 2014;6(6):394-409.

42. Zhang M, Li XM, Wang GS, et al. Thyroid cancer in systemic lupus erythematosus: a meta analysis. Int J Clin Exp Pathol. 2014;7(9):6270-3.

43. Liu X, Ji J, Forsti A, Sundquist K, Sundquist J, Hemminki K. Autoimmune disease and subsequent urological cancer. J Urol. 2013;189(6):2262-8.

44. Bernatsky S, Ramsey-Goldman R, Gordon C, Clarke AE. Prostate cancer in systemic lupus erythematosus. Int J Cancer. 2011;129(12):2966-9.

To estimate prostate cancer risk in SLE, this analysis included 6,068 male SLE patients who were observed for an average 6.3 years. The standardized incidence ratio (SIR 0.72, $95 \%$ CI $0.57-0.89$ ) showed a clear decreased risk of prostate cancer in SLE patients compared to the general population.

45. Humphrey LL, Deffebach M, Pappas M, et al. Screening for lung cancer with low-dose computed tomography: a systematic review to update the U. S. preventive services task force recommendation. Ann Intern Med. 2013;159(6):411. 